\title{
Fitness Age Score and the Risk of Long-Term Care Insurance Certification-The Kyoto-Kameoka Longitudinal Study
}

\author{
Tsukasa Yoshida ${ }^{1,2,3^{*}}$, Misaka Kimura4, Yosuke Yamada², Keiichi Yokoyama ${ }^{5,6}$, Tatsuro Ishihara7, \\ Yasuko Yoshinaka ${ }^{6}$, Aya Itoi ${ }^{8}$, Yuya Watanabe ${ }^{9}$, Noriyuki Kida ${ }^{1}$, Teruo Nomura ${ }^{1}$ \\ ${ }^{1}$ Graduate School of Science and Technology, Kyoto Institute of Technology, Kyoto, Japan \\ ${ }^{2}$ Department of Nutrition and Metabolism, National Institutes of Biomedical Innovation, Health and Nutrition, Tokyo, Japan \\ ${ }^{3}$ Senior Citizen's Welfare Section, Kameoka City Government, Kyoto, Japan \\ ${ }^{4}$ Faculty of Health and Medical Sciences, Kyoto Gakuen University, Kyoto, Japan \\ ${ }^{5}$ Non-Profit Organization Genki-Up AGE Project Inc., Kyoto, Japan \\ ${ }^{6}$ Faculty of Economics and Business Administration, Kyoto Gakuen University, Kyoto, Japan \\ ${ }^{7}$ Graduate School of Health and Sports Science, Doshisha University, Kyoto, Japan \\ ${ }^{8}$ Department of Health, Sports and Nutrition, Faculty of Health and Welfare, Kobe Women's University, Hyogo, Japan \\ ${ }^{9}$ Faculty of Health and Sports Science, Doshisha University, Kyoto, Japan \\ Email: *d5812003@edu.kit.ac.jp
}

How to cite this paper: Yoshida, T., Kimura, M., Yamada, Y., Yokoyama, K., Ishihara, T., Yoshinaka, Y., Itoi, A., Watanabe, Y., Kida, N. and Nomura, T. (2017) Fitness Age Score and the Risk of LongTerm Care Insurance Certification-The Kyoto-Kameoka Longitudinal Study. Open Journal of Epidemiology, 7, 190-200. https://doi.org/10.4236/ojepi.2017.72016

Received: April 14, 2017

Accepted: May 28, 2017

Published: May 31, 2017

Copyright (c) 2017 by authors and Scientific Research Publishing Inc. This work is licensed under the Creative Commons Attribution International License (CC BY 4.0).

http://creativecommons.org/licenses/by/4.0/ (c) (i) Open Access

\begin{abstract}
Long-Term Care Insurance System has been introduced to provide appropriate care for the elderly who needs nursing care in Japan. Physical function is one of most important function for the elderly to live independently. A previous study had established a Fitness Age Score (FAS) based on a theory for biomarker of aging. This study clarified whether the FAS could be used to predict the need for certification of long-term care (CLTC) in independent elderly people. We included 939 independent, community-dwelling-elderly (average age, 74.4 years) whose physical function was measured and for whom the CLTC was tracked for 24 months. The FAS comprised five physical fitness items: Walking time, grip strength, one-leg standing, vertical jump, and functional reach test. Based on the FAS, we calculated the odds ratio (OR) for the CLTC. Receiver operating characteristic (ROC) curves and the area under the curve (AUC) was used for further analysis. We also used the Youden Index (YI), minimum distance method (MD), and two-graph ROC method (TG) to calculate cutoff values (CVs) for screening. We divided subjects into the low and high physical fitness group with CVs. We developed cumulative CLTC curves using the Kaplan-Meier method. Sixteen people could not be tracked for the full 24 months. The measurement of FAS was completed for 798 people, of whom 28 became CLTC. The measurement of FAS was not completed for 125 people, of whom 21 became CLTC. The CLTC OR for people
\end{abstract}


with incomplete FAS was 5.553. The AUC was $0.72(\mathrm{p}<0.001)$. The CVs for the FAS were -0.29 for YI (sensitivity, $92.9 \%$ and specificity, $40.4 \%$ ), -1.23 for MD (57.1\% and $73.4 \%$ ), and -0.81 for TG (60.7\% and $60.9 \%)$. The cumulative CLTC curves according to CVs all showed significant differences ( $p=0.000$ 0.020). In conclusion, the FAS can be used to predict CLTC.

\section{Keywords}

Elderly, Physical Function, Long-Term Care, Screening, Fitness Age Score (FAS)

\section{Introduction}

Japan is an aging society in which the proportion of elderly people who are at least 65 years old has exceeded 25\%, and this proportion is expected to increase in the future. The Long-Term Care Insurance System, which began in 2000, divides certifications of needing support into two levels and certifications of needing long-term care into five levels. As the number of elderly people increases, the number of people who require long-term care is also increasing. Presently, the number of people who qualify for certification of long-term care (CLTC: including those needing support) has exceeded 6 million [1]. Therefore, early identification of those at risk of needing long-term care and appropriate intervention to improve health expectancy are important.

Improving physical fitness is a factor of improving healthy expectancy. Previous researches indicated the relationship between physical fitness and disease or mortality. Sun Q et al. traced 13,535 women who survived to at least 70 years, and "successful survivor" was defined no history of 10 major chronic diseases or coronary artery bypass graft surgery and no cognitive impairment, physical impairment, or mental health limitations. The women whose walking pace was brisk or very brisk ( $\geq 3.0 \mathrm{mph}$ ) had 2.68 fold increased odds of successful survivor compared with the women who walked easy pace $(<2.0 \mathrm{mph})$ as reference [2]. Leong DP et al. conducted longitudinal study with 139,691 men and women in 4 years, and found that hazard ratio per $5 \mathrm{~kg}$ of reduction in grip strength was 1.16 of cardiovascular mortality, 1.17 of non-cardiovascular mortality, 1.07 of myocardial infarction and 1.09 of stroke [3]. A systematic review and metaanalysis by Cooper et al. suggested that the hazard ratio of mortality comparing the lowest quarter with the highest quarter was 1.67 in grip strength, and 2.87 in walking speed [4]. In addition, Kishimoto et al. reported that highest tertile of grip strength had 0.49 of hazard ratio for all-cause mortality compared with lowest tertile in a 19-year longitudinal study with 2,527 Japanese men and women [5]. As above, there will be no more doubting that the relationship between physical fitness and disease or mortality.

Kimura et al. [6] established a Fitness Age Score (FAS) as a comprehensive, objective evaluation of fitness in elderly individuals. The FAS is a battery test 
comprising five items: the $10-\mathrm{m}$ walking test, functional reach test, one-leg standing with eyes open, vertical jumping, and grip strength. The FAS was developed using the following stepwise method: 1) a cross-sectional analysis to identify the magnitude of the association between each variable and chronical age; 2) a longitudinal analysis to identify the magnitude of true age-related changes in each variable; and 3) a stability analysis to evaluate the inter-year reliability of the annual values for each variable [6]. This stepwise method was developed by Ingram et al. and has been used to identify biomarkers of aging in humans [7]. The theoretical based methods can assess the discrepancy between chronological aging and physical fitness aging within individuals. Interestingly, grip strength and gait speed were selected as a biomarker of physical fitness aging, which are included in a definition of Fried frailty phenotype index [8] and current sarcopenia index [9].

We hypothesized that the FAS would be useful for screening elderly individuals who have a higher risk of CLTC. If the FAS was related to the future risk of CLTC, it is helpful to develop treatment plans to prevent CLTC. The purpose of this study was to clarify whether the FAS could be used to predict the need for CLTC and to design an evaluation method for that prediction.

\section{Methods}

We analyzed data from the Kyoto-KAMEOKA Study longitudinally, which is a prospective cohort study aimed at promoting and verifying the prevention of injuries and of the need for long-term care [10]. We initially evaluated 1,379 independent elderly people aged at least 65 years who attended physical function measurement clinics in Kameoka City, Kyoto Prefecture, Japan during March and April 2012. We excluded 440 people who thereafter participated in an exercise intervention for another research study [11]. The observation period was between April 2012 and March 2014, and the number of individuals who needed CLTC (including support needs) was tracked for 939 subjects for 24 months (average age 74.4 years at baseline).

The FAS [6] comprises 5 items: the10-m walking test (sec:Xa), functional reach test $(\mathrm{cm}: \mathrm{Xb})$, one-leg standing with eyes open (sec:Xc), vertical jumping $(\mathrm{cm}: \mathrm{Xd})$, and grip strength (kg:Xe); the FAS $(\mathrm{Y})$ was calculated with the following formula:

$$
\begin{gathered}
\text { Men : } \mathrm{Y}=-0.203 \mathrm{X} a+0.034 \mathrm{Xb}+0.0064 \mathrm{X} \mathrm{c}+0.044 \mathrm{Xd}+0.046 \mathrm{Xe}-3.05 \\
\text { Women }: \mathrm{Y}=-0.263 \mathrm{Xa}+0.033 \mathrm{Xb}+0.0074 \mathrm{X} \mathrm{c}+0.048 \mathrm{Xd}+0.079 \mathrm{Xe}-2.52
\end{gathered}
$$

If all 5 items of the FAS were completed, then the CLTC odds ratio was calculated.

In order to evaluate the appropriateness of CLTC screening with the FAS, we developed a receiver operating characteristic curve (ROC curve), calculated the area under the ROC curve (AUC), and evaluated the precision of the screening [12].

The cutoff value (CV) for the FAS score was created with 3 methods: 1$)$ the 
Youden Index (YI) [13] [14], which evaluates the value for which "sensitivity - (1 - specificity)" is the highest; 2) a method that evaluates the shortest distance from the coordinates (false-positive ratio, sensitivity) $=(0,1)$ to the ROC curve (the Minimum Distance method [MD]) [15]; and 3) the Two-Graph ROC method (TG) [16], which determines the value that is at the intersection of the FAS score-sensitivity curve and the FAS score-specificity curve.

For each CV that was obtained by the 3 methods, we classified subjects as having high values (high physical fitness group: High Group) or low values (low physical fitness group: Low Group), and we developed a cumulative CLTC curve using the Kaplan-Meier method [17]. We analyzed the differences between the cumulative CLTC curves for the High and Low Groups with the log rank test. Significance was set at $5 \%$ in all cases.

\section{Results}

Sixteen subjects could not be tracked for 24 months. A total of 798 subjects completed the FAS, and in 24 months, 28 of them needed CLTC. One hundred twenty-five subjects could not perform at least one of the five items; thus, they received no FAS score. Twenty-one subjects needed CLTC. The CLTC odds ratio for FAS-incomplete versus FAS-complete individuals was 5.553 (95\% confidence interval [CI], 3.04 - 10.14) (Table 1).

The AUC of the ROC curve was 0.72 (95\% CI, $0.64-0.81 ; \mathrm{p}<0.001)$ (Figure 1). The CVs for the FAS were -0.29 (sensitivity, $92.9 \%$; specificity, $40.4 \%$ ) for the YI (Figure 1), -1.23 (sensitivity, 57.1\%; specificity, 73.4\%) for MD (Figure 1), and -0.81 (sensitivity, $60.7 \%$; specificity, $60.9 \%$ ) for the TG (Figure 2).

With regard to the cumulative CLTC curves for the Low and High Groups, the YI in the Low Group was $5.14 \%$ and that in the High Group was $0.95 \%$. For the MD method, the Low Group was $7.17 \%$ and the High Group was $2.07 \%$, and for the TG method, the Low Group was 5.32\% and the High Group was 2.28\%. For the cumulative CLTC curves, a significant difference was found between the two groups for all CVs $(\mathrm{p}=0.000-0.020)$ (Figures 3-5).

\section{Discussion}

The measurement of FAS can be conducted anywhere, such as in a local community center, as long as there is enough space for a 10 - $\mathrm{m}$ walking path. We believe it is a useful method that can be applied to many people, satisfying the re-

Table 1. Subjects' classifications at the 24-month period.

\begin{tabular}{ccccc}
\hline & Independence & CLTC & Untraceable & Total \\
\hline FAS completed & 770 & 28 & 13 & 811 \\
FAS incompleted & 104 & 21 & 3 & 128 \\
Total & 874 & 49 & 16 & 939 \\
\hline
\end{tabular}

Odds ratio, 5.553 (95\% confidence interval: 3.04 - 10.14). FAS completed is the reference group, untraceable individuals were excluded. FAS: Fitness Age Score. CLTC: Certification of Long-Term Care (including support needs). 


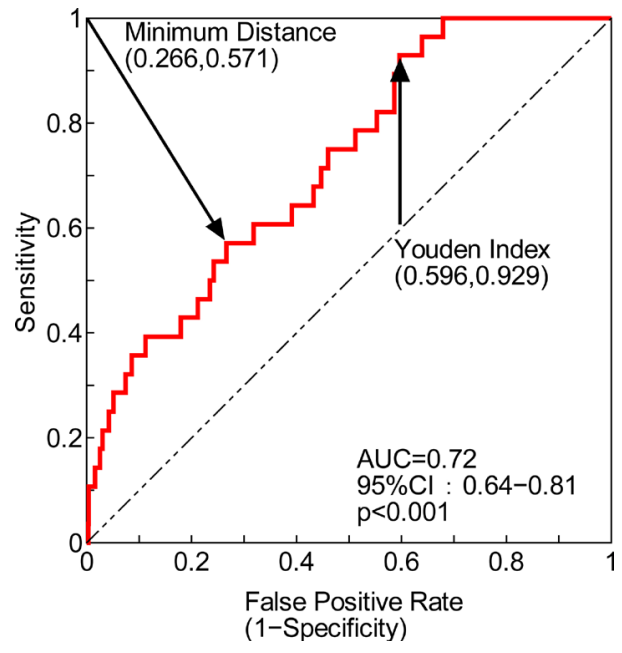

Figure 1. Receiver operating characteristic curve for the certification of long-term care (including support needed) with the fitness age score.

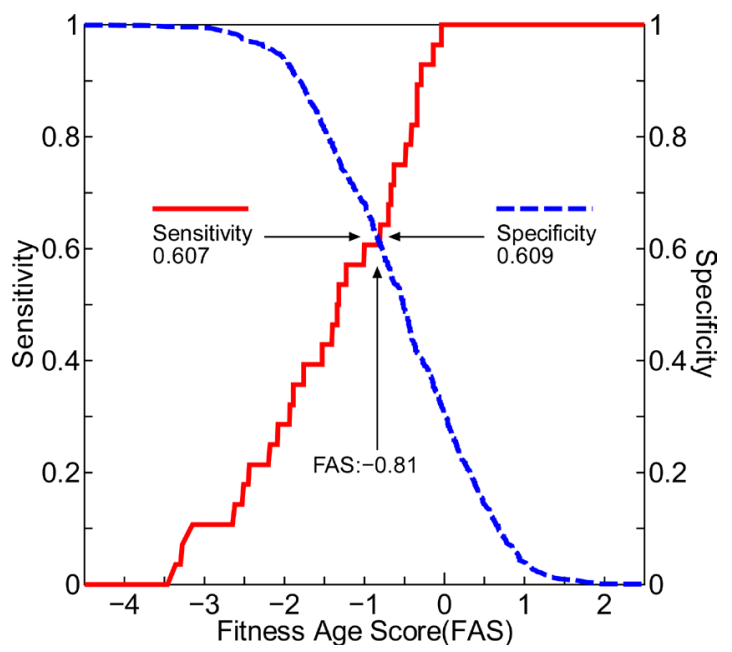

Figure 2. The relationships among sensitivity, specificity, and fitness age score with the certification of long-term care (including support needs) (two-graph receiver operating characteristic curves).

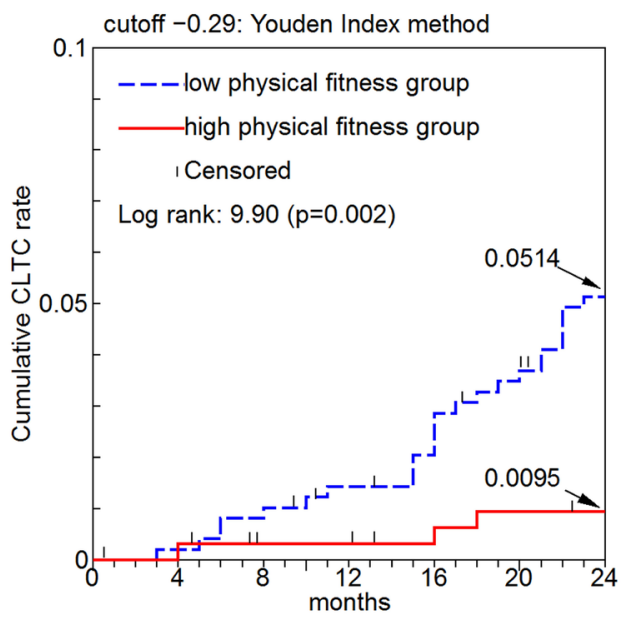

Figure 3. Cumulative certification of long-term care (CLTC) (including support needs) rate (with a fitness age score cutoff value of -0.29 ). 


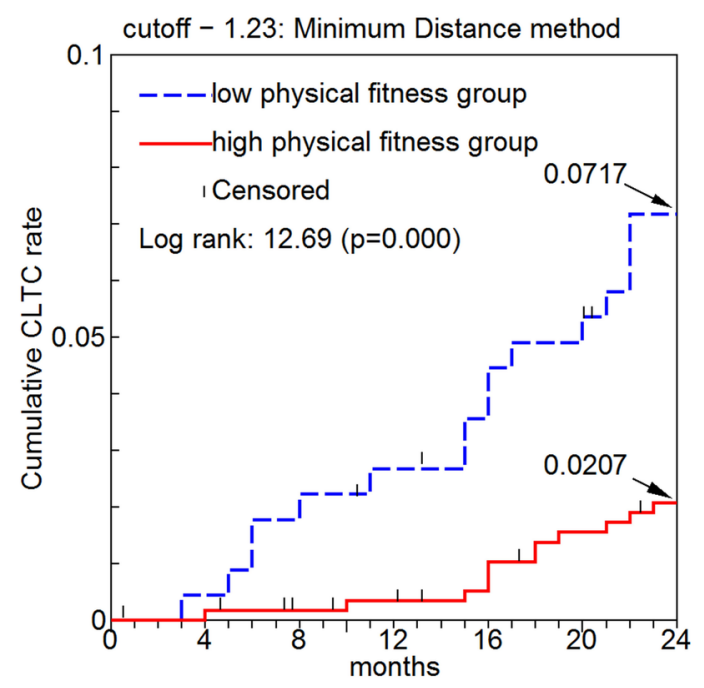

Figure 4. Cumulative certification of long-term care (CLTC) (including support needs) rate (with a fitness age score cutoff value of -1.20 ).

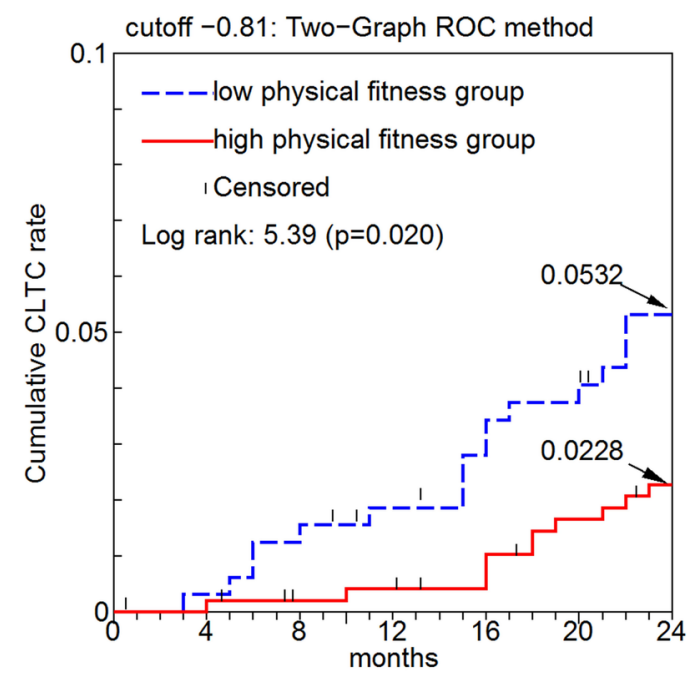

Figure 5. Cumulative certification of long-term care (CLTC) (including support needs) rate (with a fitness age score cutoff value of -0.81 ).

quirements of safety, economy, and simplicity. Safety, economy, and the performance environment should be considered when selecting functional measurement items for evaluating elderly people [18]. In addition, since field tests are often performed on a large number of people, simplicity is preferable. Therefore, using criteria that are safe and inexpensive and that can be applied in a simple way anywhere should be a condition for a field test of elderly people.

The FAS meets of all these conditions. Each item has a simple measurement method, the tester can understand the measurement method after training for a short time, and the subject being tested can safely perform it according to the instructions and support of the tester. The tools required for the measurements are a stopwatch, a tape measure, and measurement tools for grip strength and vertical jumping. These tools have recently become inexpensive and durable, and there is no need for a large initial investment or repair expense. 
People in Japan need long-term care for a variety of reasons, including diseases related to lifestyle, such as cerebrovascular disease (30\%), and problems of the locomotor system, such as joint diseases (35\%) [19]. Therefore, more than half of the causes of the need for long-term care are directly related to physical fitness or physical function.

By using the FAS to score physical fitness data, we found that the precision for CLTC screening was medium (the AUC for the ROC curve was 0.72). This result may be because the decision about whether long-term care is necessary is related to factors such as family structure and the subject's ties to the local area; that is, whether the subject's circumstances were such that appropriate support for reduced physical fitness and physical function could be obtained. Since statistically significant results were obtained with just the FAS alone, the FAS is an appropriate screening method for CLTC.

CVs for predicting CLTC were obtained by 3 methods, namely, the Youden Index (YI), the Minimum Distance method (MD), and Two-Graph ROC method (TG). The cumulative CLTC curves for the low physical fitness group and high physical fitness group, divided according to the CVs, were found to differ significantly for every CV. Therefore, the FAS could sufficiently predict CLTC within 24 months. The CVs for the three methods have different characteristics: the YI has high sensitivity, the MD has high specificity, and the CV for the TG falls between those of the other two methods.

Based on these results, we expect that these methods will be used to select measures to prevent the need for long-term care according to a person's actual situation. Recently, one method has been used to encourage physical activity by taking advantage of the local environments in which people live [20]. For example, in a local area where resources can identify many people at risk of needing long-term care and in which early intervention can be administered, we believe that wide screening using a CV based on the YI would be effective.

On the other hand, in an area that has a substantial presence of clinicians with special knowledge and training facilities but with limited screening experience, a CV obtained with the MD method would allow clinicians to identify people whose functions are greatly reduced. Even for the elderly, it is clear that muscle strength can be reinforced by administering an appropriate program [21] [22], and it is expected that the effects of preventing the need for long-term care can be improved by specific interventions.

The CLTC odds ratio was 5.553 for the FAS-incomplete group compared to the FAS-complete group. Using this result and the CVs for the FAS according to the three methods, we were able to identify a five-stage evaluation standard for the FAS. The evaluation standard was as follows: level 1: no FAS score could be measured because there were items that could not be performed; level 2: FAS is at most -1.23 ; level 3 : FAS is at most -0.80 ; level 4 : FAS is at most -0.29 ; and level 5: the FAS is over -0.29 (Table 2).

This evaluation standard can be broadly interpreted with regard to the screening results. In order to use the FAS in a practical way clinically, an evaluation standard is necessary. However, in order to create a better evaluation stan- 
Table 2. Evaluation criteria for CLTC risk by FAS.

\begin{tabular}{ccc}
\hline Evaluation & Risk & FAS \\
\hline Level 5 & Very mild & Above -0.29 \\
Level 4 & Mild & -0.81 to -0.29 \\
Level 3 & Moderate & -1.23 to -0.81 \\
Level 2 & Severe & -1.23 or less \\
Level 1 & Very severe & At least 1 item is impossible \\
\hline
\end{tabular}

FAS: Fitness Age Score. CLTC: Certification of Long-Term Care (including support needs).

dard, it is necessary to perform more detailed investigations.

\section{Limitations}

This study has two limitations. First, a detailed analysis was not performed on people for whom there were FAS items that could not be evaluated. For example, it is easy to predict that a large difference exists in physical functions between people who could perform four of the five items in the FAS and people who could only perform one item. For this reason, it is necessary to develop a revised FAS that can produce a score even if there are items in the FAS that cannot be performed and evaluated.

Second, we did not analyze the levels of CLTC in detail. In this study, all 7 levels of CLTC (including needing support) were handled as one item. Furthermore, subjects who died were excluded from the analysis. There was also no classification of the causes of CLTC. The events occurred only 10\% within 24 months. Therefore, insufficient data were obtained to perform a detailed analysis of the need for CLTC. By increasing the number of subjects or following them for a longer period of time, it might be possible to acquire the amount of data needed for making scientific judgments.

\section{Conclusion}

The purpose of this research was to clarify whether the FAS could be used to predict the need for CLTC (including support needs) for elderly people over a period of 24 months. The FAS can be used to predict the need for a CLTC with medium precision, and it was possible to calculate three types of screening CVs with different characteristics. The calculated CVs not only can be used individually, but also can be used to provide a five-stage evaluation standard for the FAS.

\section{Acknowledgements}

This research used and analyzed data from the Kyoto-KAMEOKA Study, which is a prospective cohort study for the purpose of promoting the prevention of injuries and the need for long-term care. We express our gratitude to all people related to the Kyoto-KAMEOKA Study, especially, to Mr. Matsumoto and his colleagues of Kameoka city office for their warm encouragements. We are also 
deeply grateful to all the people of Kameoka City who cooperated with our study.

This research was performed with the assistance of the Ministry of Education, Culture, Sports, Science, and Technology Grant-in-Aid for Scientific Research (A) 24240091 (Principal Investigator Misaka Kimura), the Kyoto Prefectural Regional Comprehensive Care Promotion Organization, and Kameoka City.

We would like to thank Editage (www.editage.jp) for English language editing.

\section{References}

[1] Statistic Bureau, Ministry of Internal Affairs and Communications, Japan (2016) Heisei 26 nendo Kaigo hoken jigyou joukyou houkoku nenpou [Long-Term Care Insurance Business Status Report, the Fiscal Year 2014.] (In Japanese)

http://www.e-stat.go.jp/SG1/estat/List.do?lid=000001153596

[2] Sun, Q., Townsend, M.K., Okereke, O.I., Franco, O.H., Hu, F.B. and Grodstein, F. (2010) Physical Activity at Mid-Life in Relation to Successful Survival in Women at Age 70 Years and Older. Archives of Internal Medicine, 170, 194-201. https://doi.org/10.1001/archinternmed.2009.503

[3] Leong, D.P., Teo, K.K., Rangarajan, S., Lopez-Jaramillo, P., Avezum Jr., A., Orlandini, A., Seron, P., Ahmed, S.H., Rosengren, A., Kelishadi, R., Rahman, O., Swaminathan, S., Iqbal, R., Gupta, R., Lear, S.A., Oguz, A., Yusoff, K., Zatonska, K., Chifamba, J., Igumbor, E., Mohan, V., Anjana, R.M., Gu, H., Li, W., Yusuf, S. and Prospective Urban Rural Epidemiology (PURE) Study Investigators (2015) Prognostic Value of Grip Strength: Findings from the Prospective Urban Rural Epidemiology (PURE) Study. The Lancet, 386, 266-273.

https://doi.org/10.1016/S0140-6736(14)62000-6

[4] Cooper, R., Kuh, D., Hardy, R., and Mortality Review Group; FALCon and HALCyon Study Teams (2010) Objectively Measured Physical Capability Levels and Mortality: Systematic Review and Meta-Analysis. BMJ, 341, c4467. https://doi.org/10.1136/bmj.c4467

[5] Kishimoto, H., Hata, J., Ninomiya, T., Nemeth, H., Hirakawa, Y., Yoshida, D., Kumagai, S., Kitazono, T. and Kiyohara, Y. (2014) Midlife and Late-Life Handgrip Strength and Risk of Cause-Specific Death in a General Japanese Population: The Hisayama Study. Journal of Epidemiology and Community Health, 68, 663-668. https://doi.org/10.1136/jech-2013-203611

[6] Kimura, M., Mizuta, C., Yamada, Y., Okayama, Y. and Nakamura, E. (2012) Constructing an Index of Physical Fitness Age for Japanese Elderly Based on 7-Year Longitudinal Data: Sex Differences in Estimated Physical Fitness Age. Age, 34, 203 214. https://doi.org/10.1007/s11357-011-9225-5

[7] Ingram, D.K., Nakamura, E., Smucny, D., Roth, G.S. and Lane, M.A. (2001) Strategy for Identifying Biomarkers of Aging in Long-Lived Species. Experimental Gerontology, 36, 1025-1034. https://doi.org/10.1016/S0531-5565(01)00110-3

[8] Fried, L.P., Tangen, C.M., Walston, J., Newman, A.B., Hirsch, C., Gottdiener, J., Seeman, T., Tracy, R., Kop, W.J., Burke, G. and McBurnie, M.A. for the Cardiovascular Health Study Collaborative Research Group (2001) Frailty in Older Adults: Evidence for a Phenotype. Journal of Gerontology: Medical Sciences, 56A, M146M156. https://doi.org/10.1093/gerona/56.3.m146

[9] Chen, L.K., Liu, L.K., Woo, J., Assantachai, P., Auyeung, T.W., Bahyah, K.S., Chou, M.Y., Chen, L.Y., Hsu, P.S., Krairit, O., Lee, J.S., Lee, W.J., Lee, Y., Liang, C.K., Limpawattana, P., Lin, C.S., Peng, L.N., Satake, S., Suzuki, T., Won, C.W., Wu, 
C.H., Wu, S.N., Zhang, T., Zeng, P., Akishita, M. and Arai, H. (2013) Sarcopenia in Asia: Consensus Report of the Asian Working Group for Sarcopenia. Journal of the American Medical Directors Association, 15, 95-101. https://doi.org/10.1016/j.jamda.2013.11.025

[10] Yamada, Y., Nanri, H., Watanabe, Y., Yoshida, T., Yokoyama, K., Itoi, A., Date, H., Yamaguchi, M., Miyake, M., Yamagata, E., Tamiya, H., Nishimura, M., Fujibayashi, M., Ebine, N., Yoshida, M., Kikutani, T., Yoshimura, E., Ishikawa-Takata, K., Yamada, M., Nakaya, T., Yoshinaka, Y., Fujiwara, Y., Arai, H. and Kimura, M. (2017) Prevalence of Frailty Assessed by Fried and Kihon Checklist Indexes in a Prospective Cohort Study: Design and Demographics of the Kyoto-Kameoka Longitudinal Study. Journal of the American Medical Directors Association (Accepted, In Press). https://doi.org/10.1016/j.jamda.2017.02.022

[11] Watanabe, Y., Yamada, Y., Miyake, M., Yokoyama, K. and Yoshida, T. (2014) The Establishment of Effective Intervention to Prevent Sarcopenia for Elderly. Descente Sports Science, 35, 78-86. (In Japanese)

[12] Hajian-Tilaki, K. (2013) Receiver Operating Characteristic (ROC) Curve Analysis for Medical Diagnostic Test Evaluation. Caspian Journal of Internal Medicine, 4, 627-635.

[13] Youden, W.J. (1950) Index for Rating Diagnostic Tests. Cancer, 3, 32-35. https://doi.org/10.1002/1097-0142(1950)3:1<32::AID-CNCR2820030106>3.0.CO;2$\underline{3}$

[14] Schisterman, E.F., Perkins, N.J., Liu, A. and Bondell, H. (2005) Optimal Cut-Point and Its Corresponding Youden Index to Discriminate Individuals Using Pooled Blood Samples. Epidemiology, 16, 73-81. https://doi.org/10.1097/01.ede.0000147512.81966.ba

[15] Kumar, R. and Indrayan, A. (2011) Receiver Operating Characteristic (ROC) Curve for Medical Researchers. Indian Pediatrics, 48, 277-287. https://doi.org/10.1007/s13312-011-0055-4

[16] Greiner, M., Sohr, D. and Göbel, P. (1995) A Modified ROC Analysis for the Selection of Cut-Off Values and the Definition of Intermediate Results of Serodiagnostic Tests. Journal of Immunological Methods, 11, 123-132. https://doi.org/10.1016/0022-1759(95)00121-P

[17] Smeets, M., Degryse, J., Janssens, S., Matheï, C., Wallemacq, P., Vanoverschelde, J.L., Aertgeerts, B. and Vaes, B. (2016) Diagnostic Rules and Algorithms for the Diagnosis of Non-Acute Heart Failure in Patients 80 Years of Age and Older: A Diagnostic Accuracy and Validation Study. BMJ Open, 6, e012888.

https://doi.org/10.1136/bmjopen-2016-012888

[18] Demura, S., Sohee, S. and Yamaji, S. (2008) Sex and Age Differences of Relationships among Stepping Parameters for Evaluating Dynamic Balance in the Elderly. Journal of Physiological Anthropology, 27, 207-215. https://doi.org/10.2114/jpa2.27.207

[19] Ministry of Health, Labour and Welfare, Japan (2013) Comprehensive Survey of Living Conditions, the Fiscal Year 2012. (In Japanese) http://www.mhlw.go.jp/toukei/saikin/hw/k-tyosa/k-tyosa10/4-2.html

[20] Sallis, J.F., Bowles, H.R., Bauman, A., Ainsworth, B.E., Bull, F.C., Craig, C.L., Sjöström, M., De Bourdeaudhuij, I., Lefevre, J., Matsudo, V., Matsudo, S., Macfarlane, D.J., Gomez, L.F., Inoue, S., Murase, N., Volbekiene, V., McLean, G., Carr, H., Heggebo, L.K., Tomten, H. and Bergman, P. (2009) Neighborhood Environments and Physical Activity among Adults in 11 Countries. American Journal of Preventive Medicine, 36, 484-490. https://doi.org/10.1016/j.amepre.2009.01.031 
[21] Borst, S.E. (2004) Interventions for Sarcopenia and Muscle Weakness in Older People. Age and Ageing, 33, 548-555. https://doi.org/10.1093/ageing/afh201

[22] Watanabe, Y., Tanimoto, M., Oba, N., Sanada, K., Miyachi, M. and Ishii, N. (2015) Effect of Resistance Training Using Bodyweight in the Elderly: Comparison of Resistance Exercise Movement between Slow and Normal Speed Movement. Geriatrics \& Gerontology International, 15, 1270-1277. https://doi.org/10.1111/ggi.12427

Submit or recommend next manuscript to SCIRP and we will provide best service for you:

Accepting pre-submission inquiries through Email, Facebook, LinkedIn, Twitter, etc. A wide selection of journals (inclusive of 9 subjects, more than 200 journals) Providing 24-hour high-quality service User-friendly online submission system Fair and swift peer-review system Efficient typesetting and proofreading procedure Display of the result of downloads and visits, as well as the number of cited articles Maximum dissemination of your research work

Submit your manuscript at: http://papersubmission.scirp.org/ Or contact ojepi@scirp.org 